\title{
DIFFUSION MEDIATED TRANSPORT AND THE BROWNIAN MOTOR
}

\author{
David Kinderlehrer \\ Center for Nonlinear Analysis and \\ Department of Mathematical Sciences \\ Carnegie Mellon University \\ Pittsburgh, PA USA * \\ davidk@cmu.edu
}

\begin{abstract}
We describe a dissipation principle/variational principle which may be useful in modeling motion in small viscous systems and provide brief illustrations to brownian motor or molecular rachet situations which are found in intracellular transport.

Keywords: Brownian motor, molecular rachet, motor protein, myosin, kinesin, KIF 1-A, diffusion mediated transport, mesoscopic dissipation principle, FokkerPlanck Equation, transport, Reynolds' number, Kantorovich-Wasserstein metric, weak topology, implicit scheme
\end{abstract}

\section{Introduction}

Intracellular transport in eukarya is attributed to motor proteins that transduce chemical energy into directed mechanical motion. Muscle mysosin has been known since the mid-nineteenth century and its role in muscle contraction demonstrated by A.F. Huxley and H.E. Huxley in the 1950's. Kinesins and their role in intracellular transport were discovered around 1985. These nanoscale motors tow organelles and other cargo on microtubules. They function in a highly viscous setting with overdamped dynamics; we are anticipating Reynolds' numbers about $5 \times 10^{-2}$. Taken as an ensemble, they are in configurations far from conventional notions of equlibrium even though they are in an isothermal environment. Because of the presence of significant diffusion, they are sometimes referred to as Brownian motors. Since a specific type tends

${ }^{*}$ To appear in Proc. CMDS 10. Supported in part by NSF DMS 0072194 and NSF DMS 0305794 
to move in a single direction, for example, anterograde or retrograde to the cell periphery, these proteins are sometimes referred to as molecular rachets.

Here we describe a dissipation principle that describes transport in a typical motor system, like conventional kinesin. This begins a chain of events. It suggests, in a natural way, a variational principle and an implicit scheme in the sense of Otto [14], [15] and Jordan, Kinderlehrer and Otto [9]. This determines, in turn, a system of equations analogous to that proposed by Adjari and Prost [1] or Peskin, Ermentrout, and Oster [17]. We have a clear notion of equilibrium or minimum energy for a macroscopic process, however most of the systems we meet are only metastable. It is, indeed, common to model situations in a way that this metastability disappears. Moreover, even when we are cognizant of this behavior, when we think of evolution, especially when we have a smooth solution in hand, we often neglect to think that when writing that states are close to each other, we are imposing a notion of closeness, a topology, on the dynamics. The novelty of our idea is that it sets this dynamical process in a weak topology as described by a Kantorovich-Wasserstein metric. This owes in part to a result of Brenier and Benamou, [3]. It illustrates the feasibility of mesoscale modeling for these systems.

The flashing rachet, a different type of Brownian motor, was discussed in [10]. One explanation of this was given in [2] and it has been suggested as a description of processivity in the KIF-1A family of kinesins, [12], [13]. There is a discussion in [6] as well as the Parrondo Paradox, a coin toss game somethimes thought to mimic molecular motor behavior, in $[7]$.

With a thermodynamically consistent system of differential equations in hand, we inquire of conditions that ensure transport. In the example we describe, a model for conventional kinesin, diffusion and conformational change collaborate with transport in periodic potentials. This model is highly over simplified. Asymmetry of the potentials within their period intervals is critical for transport. The origins of this asymmetry are as yet unclear but could reside in the details of the binding mechanisms of the heads to the microtuble, as well as other features.

This is a description of joint work with Michal Kowalczyk, Michel Chipot, Jean Dolbeault, and Stuart Hastings.

\section{A variational principle}

Consider an ensemble of statistically homogeneous non-interacting particles in a highly viscous medium, thought of simply as spring-massdashpots. For our setup, suppose we have probability densities $f^{*}(x)$ and 
$f(x), x \in \Omega=(0,1)$, and interpolating densities $f(x, t), x \in \Omega, 0 \leq t \leq$ $\tau$ with $f^{*}(x)=f(x, 0)$ and $f(x)=f(x, \tau)$. For this 'Eulerian' description, there is a 'Langrangian' description in terms of a family of measure preserving mappings, transfer functions, $\phi(x, t), x \in \Omega, 0 \leq t \leq \tau$ related by

$$
\int_{\Omega} \zeta(y) f(y, t) d y=\int_{\Omega} \zeta(\phi(x, t)) f^{*}(x) d x .
$$

For $f(x, t)$ there is a velocity $v(x, t)$ such that

$$
f_{t}+(v f)_{x}=0 \text { in } \Omega, 0<t<\tau \quad \text { (continuity equation) }
$$

and likewise in the 'Lagrangian' version

$$
f(\phi(x, t), t) \phi_{x}=f^{*}(x) .
$$

This is actually the Monge-Ampere Equation. They are also related by $\phi_{t}(x, t)=v(\phi(x, t), t)$.

For the ensemble of spring-mass-dashpots, the viscous dissipation moving from $f^{*}$ to $f$ via $f(x, t)$ is simply

$$
\gamma \int_{0}^{\tau} \int_{\Omega} v^{2} f d x d t
$$

for a parameter $\gamma$. When the system moves in response to a potential $\psi$, its free energy at a density $\varphi$ is

$$
F(\varphi)=\int_{\Omega}(\psi \varphi+\sigma \varphi \log \varphi) d x
$$

In this way, we arrive at a simple mesoscopic dissipation principle. The state $f$ is admissible from $f^{*}$ provided

$$
\gamma \int_{0}^{\tau} \int_{\Omega} v^{2} f d x d t+F(f) \leq F\left(f^{*}\right)
$$

for some interpolating density $f(x, t)$ with $f^{*}(x)=f(x, 0)$ and $f(x)=$ $f(x, \tau)$. We regard $\tau$ as a relaxation time. To connect this to a variational principle, we observe that [3]

$$
\frac{1}{2 \tau} d\left(f, f^{*}\right)^{2}=i n f_{A} \frac{1}{2} \int_{0}^{\tau} \int_{\Omega} v^{2} f d x d t
$$

where $A$ is the family of interpolating densities and $d$ is the KantorovichWasserstein metric defined by

$$
\begin{array}{r}
d\left(f, f^{*}\right)^{2}=i n f_{P} \int_{\Omega \times \Omega}|x-y|^{2} d p(x, y) \\
P=\text { joint distributions with marginals } f, f^{*} .
\end{array}
$$


The optimality condition for $f, v$ in $(2)$ is

$$
v_{t}+v v_{x}=0 \text { in } \Omega, 0<t<\tau \quad \text { (Burgers }{ }^{\prime} \text { Equation) }
$$

Its 'Lagrangian' form is

$$
\phi(x, t)=x+\frac{t}{\tau}(\phi(x, \tau)-x), x \in \Omega, 0<t<\tau
$$

The metric $d$ delivers the weak* topology on measures, i.e., its topology as the dual space of $C(\Omega)$, and the 'Lagrangian' form suggests that the optimality condition describes a geodesic path in this space.

For convenience we set $\gamma=\frac{1}{2}$. Our variational principle is now: given $f^{*}$, determine $f$ such that

$$
\frac{1}{2 \tau} d\left(f, f^{*}\right)^{2}+F(f)=\min
$$

The variational principle (3) provides an implicit scheme: Given $f^{(k-1)}$, set $f^{*}=f^{(k-1)}$ and determine $f^{k}$ from the minimum principle. The great merit of the Wasserstein metric is that it may be, in essence, differentiated. Thus, in the limit as $\tau \rightarrow 0$, the sequence $f^{(k)}$ tends to the solution $f$ of the standard Fokker-Planck Equation, [9], [14], [15]

$$
\begin{aligned}
& \frac{\partial f}{\partial t}=\sigma \frac{\partial^{2} f}{\partial x^{2}}+\frac{\partial}{\partial x}\left(\psi^{\prime} f\right) \quad \text { in } \quad \Omega, t>0 \\
& \sigma \frac{\partial}{\partial x} f+\psi^{\prime} f=0 \quad \text { on } \quad \partial \Omega, t>0
\end{aligned}
$$

Variational principles such as (3) above may be considered without thinking about physical systems, of course, and there is now a significant literature in this topic, and even traditional problems have unexpected interpretations, [19]. (3) firmly establishes that the coarse graining of microscopic system gives rise to weak topology dynamics at the mesoscale. For situations, like the one below, where equilibrium is never achieved, this may provide additional insight into their metastable nature. From the analysis point of view, one observes that the basic variational principle is convex and superlinear, so existence of the iterates in the implicit scheme is not usually a difficulty. Convergence as $\tau \rightarrow 0$ could be, especially for nonlinear problems.

\section{A look at conventional kinesin}

Conventional kinesin has two identical head domains (heavy chains) which walk in a hand over hand fashion along a rigid microtubule. This 
is a complicated process with a complicated transformation path comprising both the ATP hydrolysis (chemical states) and the motion (mechanical states), [8], [20]. For a crude reckoning, at a gross combinatorial level, each head is attached or in motion and is nucleotide bound or not. Assuming that a given motor has one head bound and one free at any instant leads to eight possible pathways for each cycle. We shall give a simplified description by considering the nucleotide binding and then the subsequent motion. Our dissipation/variational principle is flexible enough to accomodate this process.

As an accounting measure, divide the heads of the ensemble of motors into two sets, set 1 and set 2 ; for example, the set 1 motors bind to odd labled sites on microtubules and the set 2 motors bind to even labeled sites. This permits distance along the microtubule to be used as a process variable. Regard the conformational change and nucleotide binding to be the result of first order chemistry and the motion to be the result of ineraction with potentials, diffusion, and dissipation. Let $\rho_{1}$ and $\rho_{2}$ denote the relative densities of the set 1 and set 2 motors in the powerstroke state, when they are undergoing motion. Introduce potentials and coefficients for conformational change,

$$
\begin{aligned}
\sigma & >0 \text { constant } \\
\psi_{i} & \geq 0 \text { and } \nu_{i} \geq 0, i=1,2, \quad \text { smooth and periodic of period } \frac{1}{N}
\end{aligned}
$$

with supp $\nu_{1}=\operatorname{supp} \nu_{2}$ and $\nu_{1}+\nu_{2} \leq 1$. Let

$$
P=\mathbf{1}+\tau\left(\begin{array}{cc}
-\nu_{1} & \nu_{2} \\
\nu_{1} & -\nu_{2}
\end{array}\right)
$$

where $\tau$ is a relaxation time. Denote the free energy of this system by

$$
F(\rho)=\sum_{i=1}^{2} \int_{\Omega}\left(\psi_{i} \rho_{i}+\sigma \rho_{i} \log \rho_{i}\right) d x
$$

We may envision a cycle starting with density $\rho^{*}=\left(\rho_{1}^{*}, \rho_{2}^{*}\right)$ and proceeding by

$$
\rho^{*} \rightarrow \rho^{*} P \rightarrow \rho
$$

subject to the dissipation principle: given $\rho^{*}$ with

$$
\int_{\Omega}\left(\rho_{1}^{*}+\rho_{2}^{*}\right) d x=1 \quad \text { and } \quad \rho_{i}^{*} \geq 0 \text { in } \Omega,
$$

determine $\rho$ by

$$
\sum_{i=1}^{2} \frac{1}{2 \tau} d\left(\rho_{i},\left(\rho^{*} P\right)_{i}\right)^{2}+F(\rho)=\min
$$




$$
\int_{\Omega} \rho_{i} d x=\int_{\Omega}\left(\rho^{*} P\right)_{i} d x
$$

The variational principle (8) lucidly shows the roles of the dissipation, conformational change, and free energy in the system. It is clear that much more complex features of the process could be considered. Although there are some subtleties, (8) admits an Euler Equation which is the system [5]

$$
\begin{aligned}
& \frac{\partial \rho_{1}}{\partial t}=\frac{\partial}{\partial x}\left(\sigma \frac{\partial \rho_{1}}{\partial x}+\psi_{1}^{\prime} \rho_{1}\right)-\nu_{1} \rho_{1}+\nu_{2} \rho_{2} \text { in } \Omega, t>0 \\
& \frac{\partial \rho_{2}}{\partial t}=\frac{\partial}{\partial x}\left(\sigma \frac{\partial \rho_{2}}{\partial x}+\psi_{2}^{\prime} \rho_{2}\right)+\nu_{1} \rho_{1}-\nu_{2} \rho_{2} \text { in } \Omega, t>0 \\
& \sigma \frac{\partial \rho_{1}}{\partial x}+\psi_{1}^{\prime} \rho_{1}=0 \text { on } \partial \Omega, t>0 \\
& \sigma \frac{\partial \rho_{2}}{\partial x}+\psi_{2}^{\prime} \rho_{2}=0 \text { on } \partial \Omega, t>0 \\
& \rho_{i}(x, 0)=\rho_{i}^{0} \geq 0, \quad \text { in } \Omega, \quad i=1,2 \\
& \int_{\Omega}\left(\rho_{1}+\rho_{2}\right) d x=1
\end{aligned}
$$

and moreover this system has a solution for all time. There is, in addition, a unique stationary solution, namely the solution $\rho^{\sharp}$ of the system of ordinary differential equations [4]

$$
\begin{aligned}
& \frac{d}{d x}\left(\sigma \frac{d \rho_{1}^{\sharp}}{d x}+\psi_{1}^{\prime} \rho_{1}^{\sharp}\right)-\nu_{1} \rho_{1}^{\sharp}+\nu_{2} \rho_{2}^{\sharp}=0 \text { in } \Omega \\
& \frac{d}{d x}\left(\sigma \frac{d \rho_{2}^{\sharp}}{d x}+\psi_{2}^{\prime} \rho_{2}^{\sharp}\right)+\nu_{1} \rho_{1}^{\sharp}-\nu_{2} \rho_{2}^{\sharp}=0 \text { in } \Omega \\
& \sigma \frac{d \rho_{1}^{\sharp}}{d x}+\psi_{1}^{\prime} \rho_{1}^{\sharp}=0 \text { on } \partial \Omega \\
& \sigma \frac{d \rho_{2}^{\sharp}}{d x}+\psi_{2}^{\prime} \rho_{2}^{\sharp}=0 \text { on } \partial \Omega \\
& \int_{\Omega}\left(\rho_{1}^{\sharp}+\rho_{2}^{\sharp}\right) d x=1
\end{aligned}
$$

Note that in general $\rho^{\sharp}$ does not minimize (6).

Finally, we discuss the transport properties of $\rho^{\sharp}$. Although we have not yet untangled all details of the collaboration between diffusion and transport here, the role of asymmetry is prominent. The main idea is, writing the system above in terms of its fundamental matrix (as a first order $4 \times 4$ system) starting from $x=1$, to exploit the sign changes in 

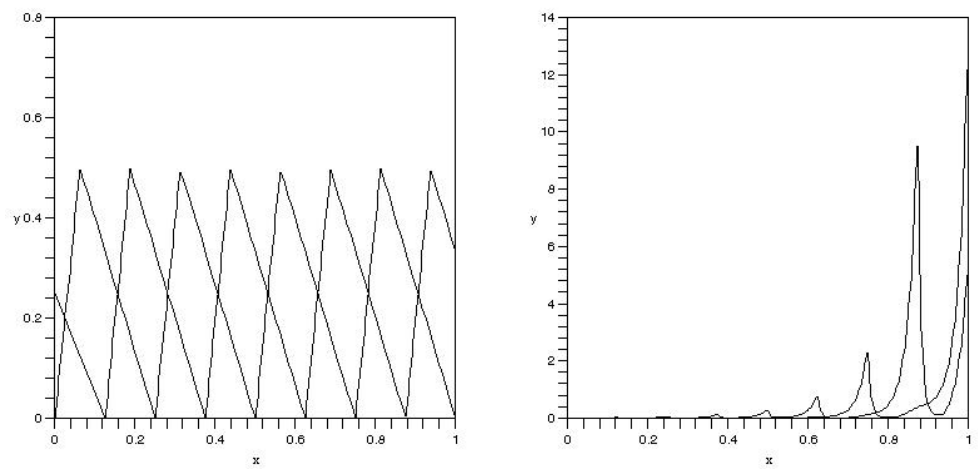

Figure 1. Interdigitated asymmetric potentials $\psi_{1}$ and $\psi_{2}$ (left) and stationary state $\rho^{\sharp}$ demonstrating about 0.9 of its mass on the right half of the interval.

$\psi_{i}^{\prime}$. For this we assume that between successive maxima, $\psi_{i}$ decreases monotonically to a unique minimum and then increases to its maximum. In this framework, our main result is that if

there is no interval where $\psi_{1}$ and $\psi_{2}$ are both increasing, and

$\nu_{1}>0$ and $\nu_{2}>0$ in $\Omega$

then

$$
\rho_{1}^{\sharp}\left(x-\frac{1}{N}\right)+\rho_{2}^{\sharp}\left(x-\frac{1}{N}\right) \leq K e^{-\frac{C}{\sigma}}\left(\rho_{1}^{\sharp}(x)+\rho_{2}^{\sharp}(x)\right), \quad x \leq 1-\frac{2}{N}
$$

At this writing, the relationship of the supports of the conformational change coefficients $\nu_{i}$ and the potentials $\psi_{i}$ is still not clear. One obvious situation where no transport can be expected is when the system (10), (11) decouples. This happens when

$$
\nu \propto\left(e^{-\frac{\psi_{2}}{\sigma}}, e^{-\frac{\psi_{1}}{\sigma}}\right)
$$

This is sometimes referred to as detailed balance, but it only concerns the balance in part of the equations. However, even in this case, retaining the $\sigma=\sigma_{0}$ above in (15)but diminishing sufficiently the diffusion coefficient $\sigma$ in (10), (11) will result in transport according to our theorem.

\section{References}

[1] Adjari, A. and Prost, J. (1992) Mouvement induit par un potentiel périodique de basse symétrie: dielectrophorese pulse, C. R. Acad. Sci. Paris t. 315, Série II, 1653. 
[2] Astumian, R.D. (1997) Thermodynamics and kinetics of a Brownian motor, Science 276 (1997), 917-922.

[3] Benamou, J.-D. and Brenier, Y. (20000 A computational fluid mechanics solution to the Monge-Kantorovich mass transfer problem, Numer. Math. 84 , 375-393.

[4] Chipot, M., Hastings, S., and Kinderlehrer, D., to appear

[5] Chipot, M., D. Kinderlehrer, D. and Kowalczyk, M. (2003) A variational principle for molecular motors, Meccanica, 38, 505-518

[6] Dolbeault, J., Kinderlehrer, D., and Kowalczyk, M. Remarks about the flashing rachet, to appear Proc. PASI 2003

[7] Heath, D., Kinderlehrer, D. and Kowalczyk, M. (2002) Discrete and continuous ratchets: from coin toss to molecular motor, Discrete and continuous dynamical systems Ser. B 2 no. 2 , 153-167.

[8] Howard, J. (2001) Mechanics of Motor Proteins and the Cytoskeleton, Sinauer Associates, Inc., 2001.

[9] Jordan, R., Kinderlehrer, D. and Otto, F. (1998) The variational formulation of the Fokker-Planck equation, SIAM J. Math. Anal. Vol. 29 no. 1, 1-17.

[10] Kinderlehrer, D. and Kowalczyk, M (2002) Diffusion-mediated transport and the flashing ratchet, Arch. Rat. Mech. Anal. 161, 149-179.

[11] Kinderlehrer, D. and Walkington, N. (1999) Approximation of parabolic equations based upon Wasserstein's variational principle, Math. Model. Numer. Anal. (M2AN) 33 no. 4, 837-852.

[12] Okada, Y. and Hirokawa, N. (1999) A processive single-headed motor: kinesin superfamily protein KIF1A, Science Vol. 283, 19

[13] Okada, Y. and Hirokawa, N. (2000) Mechanism of the single headed processivity: diffusional anchoring between the K-loop of kinesin and the $\mathrm{C}$ terminus of tubulin, Proc. Nat. Acad. Sciences 7 no. 2, 640-645.

[14] Otto, F. (1998) Dynamics of labyrinthine pattern formation: a mean field theory, Arch. Rat. Mech. Anal. 141, 63-103

[15] Otto, F. (2001) The geometry of dissipative evolution equations: the porous medium equation, Comm. PDE 26, 101-174

[16] Parmeggiani,A., Jülicher, F., Adjari, A. and Prost, J. (1999) Energy transduction of isothermal ratchets: generic aspects and specific examples close and far from equilibrium, Phys. Rev. E, 60 no. 2, 2127-2140.

[17] Peskin, C.S.. Ermentrout, G.B. and Oster, G.F. (1995) The correlation ratchet: a novel mechanism for generating directed motion by ATP hydrolysis, in Cell Mechanics and Cellular Engineering (V.C Mow et.al eds.), Springer, New York

[18] Reimann, P. (2002) Brownian motors: noisy transport far from equilibrium, Phys. Rep. 361 nos. 2-4, 57-265.

[19] Tudorascu, A. A one phase Stefan problem via Monge-Kantorovich theory,( CNA Report 03-CNA-007)

[20] Vale, R.D. and Milligan, R.A. (2000) The way things move: looking under the hood of motor proteins, Science 288, 88-95. 